\title{
Elevated Sleep Spindle Density after Learning or after Retrieval in Rats
}

\author{
Oxana Eschenko, ${ }^{1}$ Matthias Mölle, ${ }^{2}$ Jan Born, ${ }^{2}$ and Susan J. Sara ${ }^{1}$ \\ ${ }^{1}$ Department of Neuromodulation, Neuroplasticity, and Cognition, Centre National de la Recherche Scientifique, Unité Mixte de Recherche 7102, Université \\ Paris 6, 75005 Paris, France, and 2Department of Neuroendocrinology, University of Lübeck, 23538 Lübeck, Germany
}

\begin{abstract}
Non-rapid eye movement sleep has been strongly implicated in consolidation of both declarative and procedural memory in humans. Elevated sleep-spindle density in slow-wave sleep after learning has been shown recently in humans. It has been proposed that sleep spindles, 12-15 Hz oscillations superimposed on slow waves $(<1 \mathrm{~Hz})$, in concert with high-frequency hippocampal sharp waves/ripples, promote neural plasticity underlying remote memory formation. The present study reports the first indication of learning-associated increase in spindle density in the rat, providing an animal model to study the role of brain oscillations in memory consolidation during sleep. An odor-reward association task, analogous in many respects to human paired-associate learning, is rapidly learned and leads to robust memory in rats. Rats learned the task over 10 massed trials within a single session, and EEG was monitored for $3 \mathrm{~h}$ after learning. Learning-induced increase in spindle density is reliably reproduced in rats in two different learning situations, differing primarily in the behavioral component of the task. This increase in spindle density is also present after reactivation of remote memory and in situations when memory update is required; it is not observed after noncontingent exposure to reward and training context. The latter results substantially extend findings in humans. The magnitude of increase $(\sim 25 \%)$ and the time window of maximal effect $(\sim 1 \mathrm{~h}$ after sleep onset) were remarkably similar to human data, making this a valid rodent model to study network interactions through the use of simultaneous unit recordings and local field potentials during postlearning sleep.
\end{abstract}

Key words: spindles; slow-wave sleep; memory consolidation; retrieval; off-line processing; rats

\section{Introduction}

Memory consolidation is a long-lasting, active process that necessarily involves interactions among brain systems to organize, update, or strengthen existing memories (Dudai, 2004; Sara, 2006). There is a large body of literature, dating from the 1960 s to 1970 s, generated from human and animal studies, indicating that these consolidation processes continue off-line during sleep (for review, see Smith, 1985). A recent surge of rigorous studies in humans reaffirms the beneficial effects of sleep on memory. Previous studies had focused on the rapid eye movement (REM) phase; slow-wave sleep (SWS) has been strongly implicated now, as well (for review, see Gais and Born, 2004; Rauchs et al., 2005; Walker and Stickgold, 2006). Sleep spindles, 12-15 Hz oscillations superimposed on slow waves (Molle et al., 2002), may promote neural plasticity underlying learning and memory (Steriade and Timofeev, 2003; Rosanova and Ulrich, 2005). Moreover, there is recent evidence for an increase in spindle density after verbal learning (Gais et al., 2002; Clemens et al., 2005) or motor

Received July 25, 2006; revised 0ct. 25, 2006; accepted 0ct. 26, 2006.

This work was supported by Volkswagenstifftung to S.J.S. and J.B. Julien Lelong performed the behavioral training, and Jacques Fuzellier provided engineering support. We thank Dr. Wiâm Ramadan for critical reading of this manuscript.

Correspondence should be addressed to Dr. Susan J. Sara, Centre National de la Recherche Scientifique, Unité Mixte de Recherche 7152, College de France, 75005 Paris, France. E-mail: sjsara@ccr.jussieu.fr.

D0I:10.1523/JNEUROSCI.3175-06.2006

Copyright $\odot 2006$ Society for Neuroscience $\quad$ 0270-6474/06/2612914-07\$15.00/0 learning (Smith and MacNeill, 1994; Meier-Koll et al., 1999; Fogel et al., 2002).

Interest in SWS in memory consolidation is additionally reinforced by rodent studies reporting experience-driven replay of neuronal ensembles in various brain regions during this sleep stage (Pavlides and Winson, 1989; Wilson and McNaughton, 1994; Skaggs and McNaughton, 1996; Qin et al., 1997; Sutherland and McNaughton, 2000; Hirase et al., 2001). This experiencerelated reactivation of hippocampal neurons occurs during highfrequency hippocampal oscillations known as sharp waves/ripples (Kudrimoti et al., 1999; Foster and Wilson, 2006). Sharp waves/ripples in the hippocampus were first described by Buzsaki (1989), who proposed that such activity should promote plasticity in hippocampal-cortical afferents, necessary for memory formation. Data from several laboratories, including our own, show that hippocampal ripples are temporally related to cortical spindles and both are grouped by slow oscillations (Siapas and Wilson, 1998; Sirota et al., 2003; Moelle et al., 2006), providing the first glimpse of possible mechanisms underlying a hypothesized cortical-hippocampal information transfer occurring during SWS.

In pioneering studies, the replay of neuronal ensembles was interpreted as off-line memory reactivation. The data were obtained from overtrained animals performing a highly stereotyped behavior or after exploratory behavior, precluding precise characterization of memory content. On the other hand, human studies that provide convincing evidence of beneficial effects of sleep 
on learning and memory remain highly speculative in terms of brain-mediating mechanisms because of obvious methodological limitations. Thus, there is a need to bridge animal and human approaches to fully understand the contribution of sleep to memory formation.

The present study in rats was inspired by recent human studies demonstrating elevated spindle density during non-REM sleep after various versions of paired-associate or motor-skill learning (Fogel et al., 2002; Gais et al., 2002; Clemens et al., 2005). The relationship between memory processing and spindle density during SWS has not yet been demonstrated in nonhumans. Odor-reward association, analogous in many respects to human paired-associate learning, was used to reveal a learning-related increase in spindle density. This increase also occurred after reactivation of remote memories and in situations when memory update was required.

\section{Materials and Methods}

Animals. Male Sprague Dawley rats (Charles River Laboratories, Le Genest St. Isle, France) weighting 350-400 g were used ( $n=49)$. The rats were housed individually, handled daily, and kept on a $12 \mathrm{~h}$ light/dark cycle with lights off at 8:00 P.M. Water and food were available ad libitum. All procedures were performed following the 1986 European Communities Council Directive and Ministère de l'Agriculture et de la Foret, Commission Nationale de l'Experimentation Animal decree 87848 (France).

Surgery and electrophysiological recordings. Animals were anesthetized with sodium pentobarbital ( $40 \mathrm{mg} / \mathrm{kg}$, i.p., initial dose with $0.1 \mathrm{cc}$ supplements given as necessary) and fixed in a stereotaxic frame. Atropine sulfate $(0.2 \mathrm{mg} / \mathrm{kg})$ was administered to minimize respiratory distress. The skull was exposed, and burr holes were made for the placement of the stainless steel skull screws used for subdural EEG recordings. Two screw electrodes were placed over the left prefrontal cortex [anteroposterior (AP), +4.0; lateral (L), 0.5; reference: AP, +0.5; L, 0.5]. Another skull screw served as ground. Electromyogram (EMG) activity was recorded from the dorsal neck muscles with insulated multistranded wires.

Recording was performed in a box $(25 \times 25 \times 50 \mathrm{~cm}$ in size $)$. The rat was connected to the amplifier by a cable allowing free movement within the box. Behavior was tracked by a video camera (Quickcam; Logitech, Moulin du Choc, Switzerland) mounted on the top of the recording box. The video image was synchronized with electrophysiological recordings. Recordings lasted for up to $3 \mathrm{~h}$ between 10:00 A.M. and 6:00 P.M. during the light period (i.e., when rats spend most of their time sleeping).

EEG and EMG activity were recorded continuously and digitized using a CED Power 1401 converter and Spike2 software (Cambridge Electronic Design, Cambridge, UK). EEG signals were amplified (1000×) and filtered between 0.01 and $500 \mathrm{~Hz}$ (model 1700; A-M Systems, Carlsborg, WA). EMG was amplified $(1000 \times)$ and filtered at $30-500 \mathrm{~Hz}$. The signals were sampled at $2 \mathrm{kHz}$. Data were stored on a computer for off-line analysis.

Behavioral procedures. After a 1 week recovery from surgery, rats were put on a food-restricted diet ( $20 \mathrm{~g}$ per day; body weight $\geq 80 \%$ of freefood weight) and habituated to the recording box and the plugging procedure to ensure that they behave naturally and spend sufficient time sleeping. Then a baseline activity was recorded for $\geq 3 \mathrm{~h}$. In experiment 1 , on the day after the baseline recording, an animal was trained either on the three-way odor-reward association "nose-poke" task $(n=9)$ or fourway odor-reward association "digging" task $(n=16)$. The nose-poke task has been used in our laboratory for rapid appetitive learning in rats and was described in detail previously (Tronel and Sara, 2002). Briefly, three sponges with a hole in the middle and impregnated with different odors were placed in the corners of the experimental box $(1 \times 1 \times 1 \mathrm{~m})$. The reward (chocolate rice cereal; Kellogg's, Battle Creek, MI) was placed at the bottom of the hole of the sponge with the designated target odor. A nose poke into the hole obtained reward. The digging task was a slightly modified version of the odor-reward sponge task differing mainly in the behavioral response required. For rats assigned to this task, digging be- havior was progressively shaped until animals could retrieve reward located on the bottom of the gravel-filled cup. Four cups filled with gravel, each containing a powdered spice with a distinct odor, were placed in the corners of the experimental box. The location of the target odors in both training conditions varied randomly across trials. Rats were allowed 5-10 massed trials with a 3 min cutoff time and a 1-2 min intertrial interval to make an odor-reward association (total training duration, $\sim 30 \mathrm{~min}$ ).

In experiment 2 , an additional group of rats $(n=9)$ was first trained on the digging task and submitted to surgery 1-2 weeks later. After the recovery period, rats were re-exposed to the learning situation $10-30 \mathrm{~d}$ after the initial training. Rats were allowed five trials to test their remotememory retention, after which they were placed in the recording chamber for EEG monitoring.

In experiment 3 , a subgroup of rats used in experiment $1(n=10)$ was subjected to each of the following four test situations: baseline, learning, retrieval, and extinction, which were performed on 4 consecutive days. The learning and retrieval tests were similar to experiments 1 and 2. For the extinction test, the reward was not available on any trial.

In experiment 4 , on the day after the baseline recording, rats $(n=7)$ were allowed to explore a novel environment (training box) with the reward randomly distributed over the floor. No specific odors were present in the box.

All behavioral and recording tests were performed at the same time of the day for an individual rat to avoid a potential circadian rhythm effect.

Data processing and statistical analysis. Learning was characterized in terms of the latency for a correct response and number of choice errors before finding the reward. Electrophysiological data processing was performed using Spike2 software or custom software based on the built-in script language (Cambridge Electronic Design). Power spectra of delta $(0-4 \mathrm{~Hz})$, theta $(5-10 \mathrm{~Hz})$, and spindle $(12-15 \mathrm{~Hz})$ frequency bands were calculated continuously, and sleep-wake episodes were scored by visual assessment for $10 \mathrm{~s}$ epochs according to the standard criteria (Bjorvatn et al., 1998). Briefly, the awake state was marked by the presence of low-amplitude fast activity associated with EMG tonus; SWS was identified by continuous high-amplitude slow activity, regular appearance of spindles, and decreased EMG activity. Transitions from SWS into REM sleep were identified by a decrease in high-amplitude slow activity, increase of theta activity, and presence of spindles. REM sleep was characterized by dominant theta activity, low-voltage fast activity, and an absence of EMG tonus. Behavioral states were additionally verified by video recording.

To identify spindles, a low-pass finite-impulse response (FIR) filter of $30 \mathrm{~Hz}$ was first applied on the EEG signal, and the resulting signal was down sampled to $200 \mathrm{~Hz}$. Subsequently, a FIR bandpass filter of 12-15 $\mathrm{Hz}$ was used. After bandpass filtering, the root mean square (rms) was calculated at every time point using a time window of $0.1 \mathrm{~s}$, and finally, the rms signal was smoothed with a moving average of this signal. The automatic spindle detection algorithm was applied to the $\sigma \mathrm{rms}$ signal. To take into account considerable differences in EEG signal amplitude, a threshold of 2 SDs of the $\sigma$ rms signal during SWS episodes was calculated for each recording. The time stamps marked all of the threshold crossings of the $\sigma$ rms signal; an upward-rising crossing defined the spindle onset, and the next falling crossing defined the end of the spindle. The minimal spindle duration was limited to $0.5 \mathrm{~s}$. Reliability of the spindle-detection algorithm was verified by visual observation. The spindle density was calculated over $1 \mathrm{~min}$ intervals. Additionally, a low-pass filter with an FIR filter of $3.5 \mathrm{~Hz}$ was applied to the EEG to produce the slow oscillation (delta) signal. To estimate the amplitude of slow waves, the rms was calculated at every time point using a time window of $1 \mathrm{~s}$, and the rms signal was smoothed over $10 \mathrm{~s}$ time intervals. The theta/delta ratio was also calculated for each $10 \mathrm{~s}$ interval to indicate the dominant presence of theta rhythm during REM sleep. The analysis was restricted to SWS episodes with continuous spindling activity ( $\geq 1$ spindle per $10 \mathrm{~s}$ ), a sleep state closely analogous to human non-REM sleep stage 2 (De Gennaro and Ferrara, 2003).

Because the temporal pattern of sleep episode onset and duration varied greatly between individual rats and across experimental conditions, all sleep indices were averaged over 30 min time intervals for parametric analysis. Repeated-measures ANOVA was used for between- 
group (experiments 1 and 2) and between-training (experiment 3) condition comparisons.

\section{Results}

Sleep spindles are readily detected in the rat EEG and serve as indicators of SWS. The results of a preliminary study, in which sleep was monitored for up to $6 \mathrm{~h}$ after learning, indicated that the most robust effect on the learning-modulated sleep spindle density was observed during the first hour of postlearning SWS (supplemental material, available at www.jneurosci.org). Taking this observation into account, the results described below are limited to $2 \mathrm{~h}$ of postexperience SWS monitoring.

\section{Experiment 1: effects of training condition}

To eliminate a possible contribution of the quality of the odors or motor component of an odor-reward associate learning, rats were tested on either nose-poke $(n=9)$ or digging $(n=16)$ versions of an odor-discrimination task (see Materials and Methods for task description). Both versions of the task are easily acquired by most rats within three to five massed trials (supplemental Fig. 2, available at www.jneurosci.org as supplemental material). The day before behavioral manipulation, a baseline sleep was recorded in each rat at the same time of day. Repeatedmeasures ANOVA with TIME (four 30 min intervals) and DAY of testing (baseline or learning) as repeated measures and TASK (nose poke or digging) as a between-group factor revealed a significant DAY effect $\left(F_{(1,23)}=15.36 ; p=0.001\right)$ and TIME $\times$ DAY interaction $\left(F_{(3,21)}=11.13 ; p<0.001\right)$ because of an elevated spindle density $1 \mathrm{~h}$ after learning (Fig. 1$)$. There was no TIME $\times$ $\operatorname{TASK}\left(F_{(3,21)}=0.49 ; \mathrm{NS}\right)$ or DAY $\times \operatorname{TASK}\left(F_{(1,23)}=0.14 ; \mathrm{NS}\right)$ interaction, indicating that the learning effect was present after both types of training conditions. The range and the average degree of change were comparable between the groups. The postlearning $1 \mathrm{~h}$ increase in spindle density was not accompanied by a change in the spindle duration in either group of rats.

The absolute amplitude of the spindle frequency band (12-15 $\mathrm{Hz}$ ) displayed a typical temporal pattern, decreasing over time $\left(F_{(3,21)}=8.37 ; p=0.001\right)$. However, the amplitude was slightly but significantly elevated during the first SWS episodes after learning (TIME $\times$ DAY interaction: $F_{(3,21)}=3.22 ; p<0.05$ ), when the spindle number was at its highest. This effect did not depend on the type of learning. The other sleep parameters analyzed (the amplitude of delta oscillations, total SWS duration, and SWS onset) had neither task-specific differences nor learning-dependent effects (Table 1). The temporal dynamic of the amplitude of delta oscillations was typical for a normal sleep pattern, decreasing over time $\left(F_{(3,21)}=11.80 ; p<0.001\right)$ regardless of the particular behavioral condition (TIME $\times$ TASK: $F_{(3,21)}=$ 1.91 ; NS). More details about the general sleep characteristics are given in the supplemental Results (available at www.jneurosci. org as supplemental material).

Thus, the increase in spindle density during SWS immediately after learning did not depend on a particular sensory experience or procedural component of the odor-reward association. The effect was limited to a $1 \mathrm{~h}$ time window during which sleep spindles occurred more frequently. There were no other detectable changes in sleep characteristics for $\geq 2 \mathrm{~h}$ after learning.

Experiment 2: effect of memory retrieval on rat sleep pattern To compare the sleep pattern after initial acquisition and after retrieval of a well established memory, rats from experiment 1 , which were exposed to a learning situation for the first time (learning group, $n=25$ ), were compared with rats that were

\section{Digging Task}
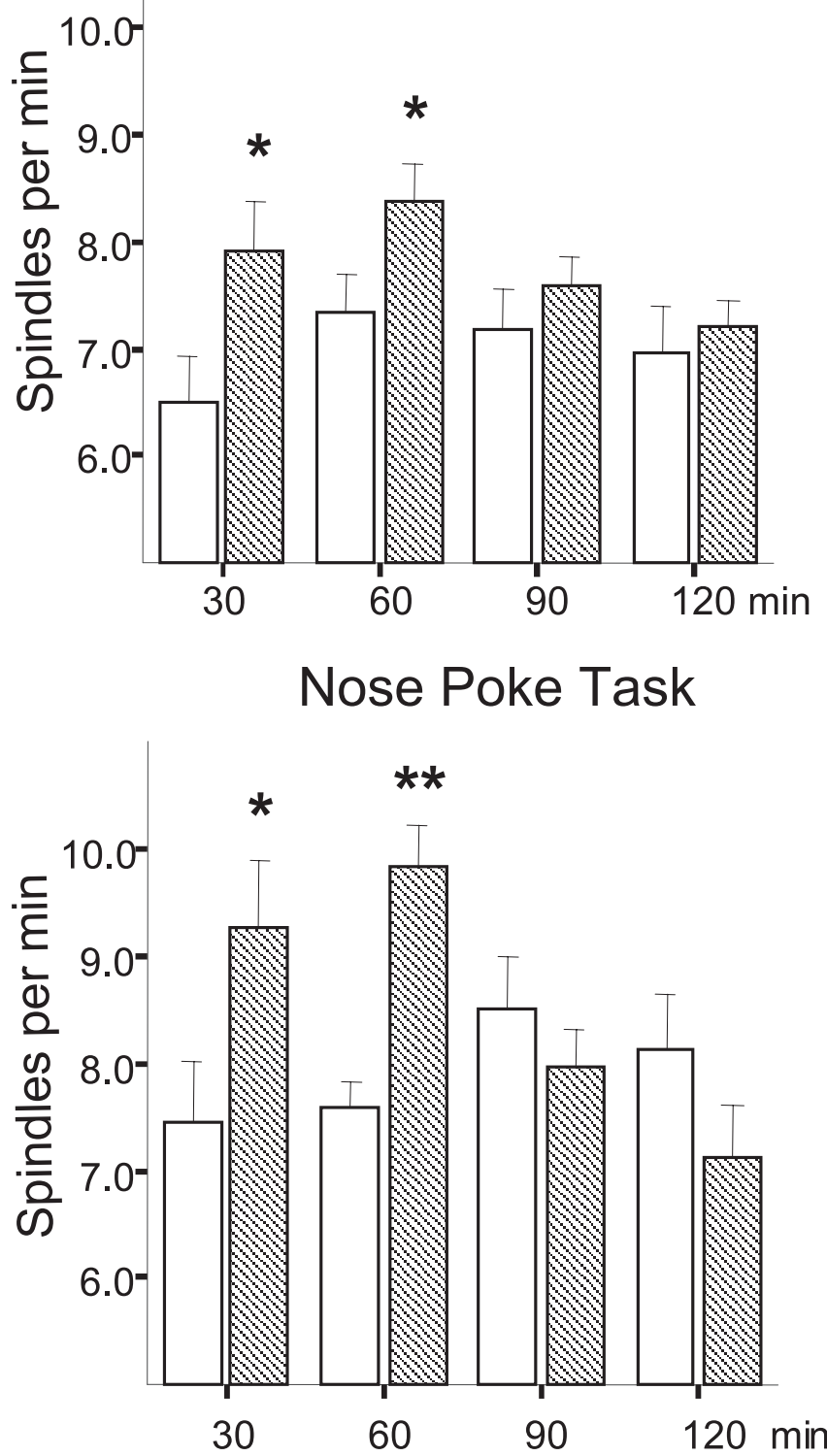

Figure 1. Spindle density across $2 \mathrm{~h}$ of recording before and after learning of a digging (top) or nose-poke (bottom) odor-reward association task. The time from SWS onset is indicated. Bars represent an average spindle density for consecutive 30 min time intervals. Open bars, Baseline recording; hatched bars, postlearning recording. ${ }^{*} p<0.05,{ }^{* *} p<0.01$ for betweencondition orthogonal comparisons (Newman-Keuls test).

trained on the digging version of the odor-reward association task and tested again in the same training condition after an extended interval (10-30 d; retrieval group, $n=9$ ). Because there were no task-specific differences found for the learning group (see experiment 1), data from both training conditions (digging and nose poke) were combined. An additional independent control group $(n=15)$, consisting of rats not submitted to any learning situation, allowed for between-group analysis. Baseline sleep was recorded in each rat; the next day, at the same time of day, a behavioral test (learning or retrieval) preceded the sleep recording; baseline sleep was recorded on 2 consecutive days in the control group. Given the results of experiment 1, the spindle density analysis was limited to the first hour after SWS onset.

Figure 2 summarizes the results of this experiment. Spindle 
Table 1. Baseline and postlearning parameters of SWS during the $1 \mathrm{~h}$ after sleep onset (mean \pm SEM)

\begin{tabular}{|c|c|c|c|c|}
\hline & \multicolumn{2}{|l|}{ Digging task } & \multicolumn{2}{|l|}{ Sponge task } \\
\hline & Baseline & Learning & Baseline & Learning \\
\hline Spindles per minute & $6.9 \pm 0.3$ & $8.1 \pm 0.4^{* *}$ & $7.5 \pm 0.4$ & $9.6 \pm 0.4^{* *}$ \\
\hline Spindle duration (s) & $0.83 \pm 0.01$ & $0.81 \pm 0.01$ & $0.82 \pm 0.02$ & $0.82 \pm 0.01$ \\
\hline Spindle band $(\mu \mathrm{V}), 12-15 \mathrm{~Hz}$ & $17.0 \pm 1.1$ & $18.0 \pm 1.1^{* *}$ & $18.8 \pm 3.7$ & $20.2 \pm 4.0^{*}$ \\
\hline Delta band $(\mu \mathrm{V}), 0-4 \mathrm{~Hz}$ & $49.1 \pm 3.3$ & $53.0 \pm 3.5$ & $43.9 \pm 7.7$ & $44.7 \pm 7.5$ \\
\hline Total SWS (min) & $12.0 \pm 1.1$ & $12.6 \pm 0.8$ & $11.4 \pm 1.4$ & $13.2 \pm 1.2$ \\
\hline SWS onset (min) & $30.0 \pm 2.8$ & $23.5 \pm 3.7$ & $13.7 \pm 2.8$ & $13.2 \pm 4.1$ \\
\hline
\end{tabular}

${ }^{*} p<0.05$ and ${ }^{* *} p<0.01$ for within-group paired comparisons. day of recording. However, this effect did not seem to be related to a particular behavioral experience because it was also observed in the control (nonlearning) condition (DAY $\times$ BEH_MANIP interaction: $F_{(2,46)}=0.56$; NS); rather, a longer SWS was related to a shorter SWS onset on the second recording day in all groups $\left(F_{(1,46)}\right.$ $=11.25 ; p<0.01)$.

\section{Experiment 3: multiple learning experience}

This experiment was a within-animal design to investigate the

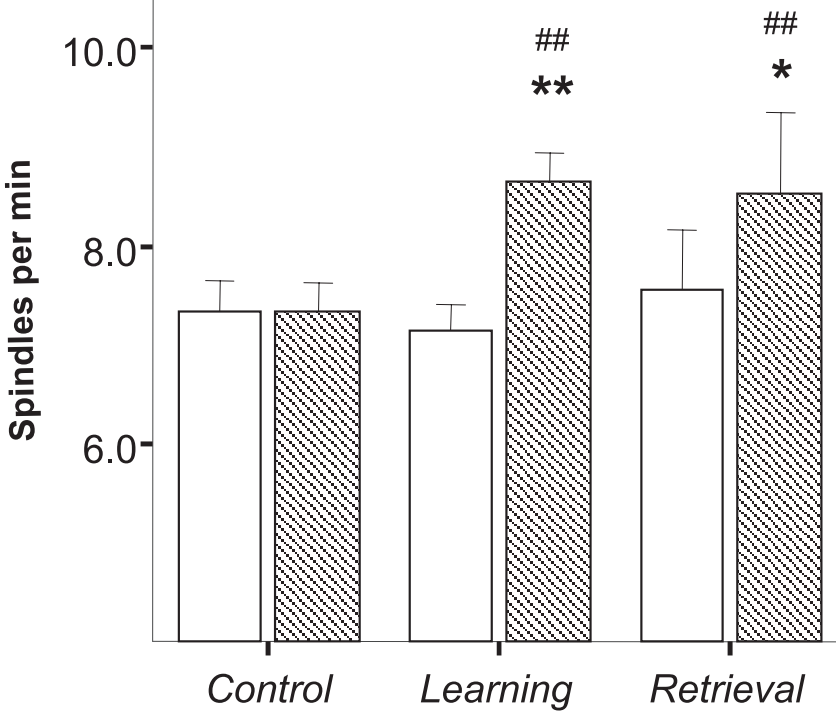

Figure 2. Spindle density (mean $\pm \mathrm{SEM}$ ) as a function of the type of memory manipulation (experiment 2, new vs old memory). Bars represent average spindle density during the first hour after SWS onset. Open bars, Baseline (nonmanipulated) sleep recording for all groups; hatched bars, repeated sleep recording. Control, Baseline (nonlearning) recordings on 2 successive days; Learning, baseline (open bar), then sleep after exposure to a new learning situation for the first time; Retrieval, baseline (open bar), then sleep after a new exposure to the learning situation several days after the initial learning. ${ }^{*} p<0.05,{ }^{* *} p<0.01$ for within-group comparisons; $\# p<0.01$ comparison with the control group.

density on the baseline day and after behavioral manipulation (learning or retrieval) is shown for each group. A similar increase in spindle density was observed after both new learning and retrieval of old memories. Two-way ANOVA with DAY as a repeated measure and a type of behavioral manipulation $(\mathrm{BEH}-$ _MANIP) as the between-group factor confirmed the above observation by revealing a significant DAY effect $\left(F_{(1,46)}=22.49\right.$; $p<0.001)$ and DAY $\times$ BEH_MANIP interaction $\left(F_{(2,46)}=8.26\right.$; $p=0.001$ ). Subsequent post hoc comparisons (Newman-Keuls test) indicated that the effect was as a result of an elevated spindle density after both learning and retrieval, whereas the spindle density did not differ between two recordings in the control group (see Fig. 2 for pairwise comparisons). The duration of individual spindles did not differ between $2 \mathrm{~d}$ of testing in any of the experimental groups $\left(F_{(1,46)}=3.68\right.$; NS); this result confirmed that the effect on spindle density was primarily related to the number of spindle events occurring during SWS after learning or memory reactivation.

There were no between- or within-group differences found in the amplitude of delta oscillations. The total SWS duration over the period of elevated spindle density (the first hour after SWS onset) was slightly longer $\left(F_{(1,46)}=4.21 ; p<0.05\right)$ on the second level of postexperience sleep-spindle density in a condition of continuously changing information about the environment, presumably involving a frequent (daily) memory update (a situation most common in real life). Rats $(n=10)$ were subjected to a series of daily tests that differed in information and/or memory content: (1) acquisition of new information (learning as in experiment 1), (2) re-exposure identical to the learning situation (retrieval as in experiment 2, but with a 24 h delay), and (3) reexposure to a modified learning situation (absence of expected reward; extinction). Spindle density was compared with the baseline SWS level (without any behavioral experience preceding sleep) recorded on the day before the behavioral testing, at the same time of day.

Repeated-measures ANOVA with DAY of test (baseline, learning, retrieval, and extinction) as a repeated measure revealed a significant DAY effect $\left(F_{(3,27)}=3.52 ; p<0.05\right)$. The post hoc comparisons (Fisher's least significant difference) confirmed that the spindle density was elevated compared with the baseline for all three test situations (Fig. 3, top). The range and the degree of change were comparable across conditions (Fig. 3, bottom). There was no difference in the other sleep parameters (spindle duration, SWS onset time, SWS duration, delta power).

\section{Experiment 4: effect of nonspecific exploration on spindle density}

To control for nonspecific effects of exploratory activity and reward, a group of rats $(n=7)$ was submitted to baseline recording and the next day explored the experimental box with randomly available reward, followed by a $2 \mathrm{~h}$ recording session. The spindle density after simple exploration and free-reward consumption did not differ from that during the baseline condition, when rats were transferred to the recording box directly from the home cage $\left(F_{(1,6)}=0.17\right.$; NS) (Fig. 4). Thus, an increase in spindle density was restricted to conditions of odor-reward learning.

\section{Discussion}

The learning-dependent activation of corticothalamic networks, expressed as an increase in spindle density during SWS, has been described in humans by several investigators (Meier-Koll et al., 1999; Fogel et al., 2002; Gais et al., 2002; Clemens et al., 2005). The present study is the first demonstration of the same phenomenon in rats and substantially extends findings in humans. The result was reliably replicated in two different odor-reward association tasks that differed in the nature of the sensory information (type of odorants) and a behavioral component of the learning (nose poke or digging for reward). Moreover, the study was extended to include reactivated remote memories (up to $30 \mathrm{~d}$ delayed memory retention test) and extinction, a situation requiring memory update concerning response-reinforcement contingencies. The study used both within- and between-animal 


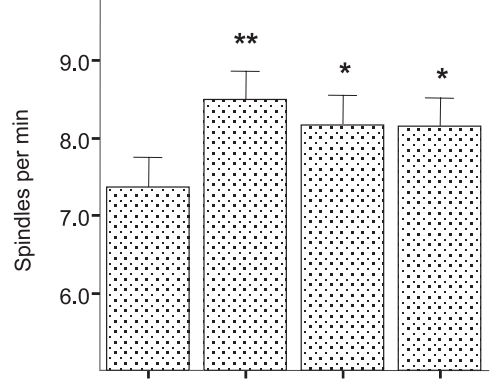

Baseline Learning Retrieval Extinction

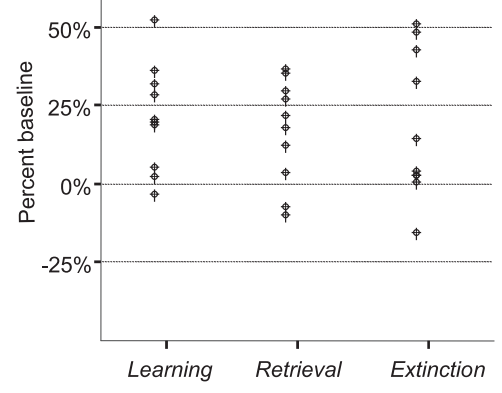

Figure 3. Spindle density (mean \pm SEM) after multiple exposure to different learning situations (experiment 3 , content of memory manipulation). Top, Bars represent average spindle density during the first hour after SWS onset during four test situations presented in a daily sequence, as follows: Baseline; Learning, sleep after exposure to a new learning situation for the first time; Retrieval, sleep after repeated exposure to the identical learning situation; Extinction, sleep after exposure to the learning context with no reward. ${ }^{*} p<0.05$, ${ }^{* *} p<0.01$ for comparison with the baseline. Bottom, Dots represent the percentage of baseline increases for each individual observation. Note that spindle numbers after retrieval and extinction did not differ from those after learning ( $p>0.05$ ).

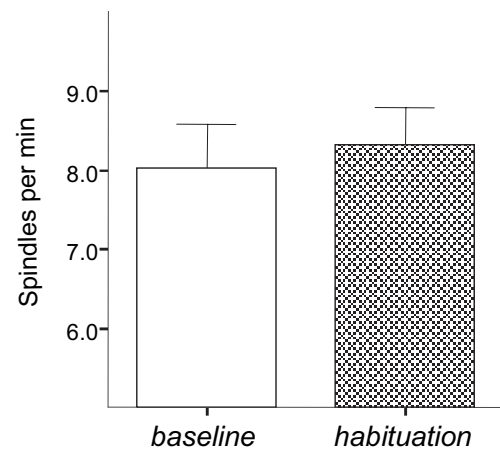

Figure 4. Average spindle density during the first hour after SWS onset during baseline (nonmanipulated; open bar) sleep recording and after a 10 min exploration of a novel environment with freely distributed reward (hatched bars) on a following day. Note that there was no elevated spindle density observed after a simple exploration of a novel situation not requiring odor-reward association. Bars represent mean \pm SEM.

designs, showing that not only was there a significant increase in spindle density after training compared with a baseline recorded on the previous day, but there was a significant difference in spindle density in trained rats compared with nontrained controls submitted to successive recording sessions (experiment 2). This increase in spindle density did not occur in animals that were submitted to a session of habituation, involving exploration of the novel experimental environment and exposure to free "reward" (experiment 4). Nevertheless, although increase in spindle density was only seen after the animal had been involved in formal odor-reward association learning, there was no direct corre- lation between the sleep spindle density after learning and the subsequent behavioral performance. This could be because of the relative facility with which the task is learned. Rats rarely make a choice error after the first two trials, although their performance further improves in terms of latency (Tronel and Sara, 2002). Only a few human studies have reported a correlation between the actual spindle density and acquisition rate (Gais et al., 2002) or spindle density and retention (Clemens et al., 2005), whereas others relate spindle density merely to the subject's cognitive abilities regardless of whether learning occurred before sleep (Schabus et al., 2004).

Striking similarities to results described in human studies were found in the present experiments. The effect was most pronounced during the first hour of postlearning sleep, which compares well to the learning effects reported for the first $90 \mathrm{~min}$ sleep cycle in humans (Gais et al., 2002; Huber et al., 2004). Moreover, the average magnitude of the increase was similar, $\sim 25 \%$ in rats and 33.5\% in humans (Gais et al., 2002). Considerable individual differences were observed in both human and rat studies, as illustrated in Figure 5, with a few individuals in each species showing learning without the sleep changes. It is possible that in these cases, the effects might have occurred outside of the time window investigated. Finally, the effect was restricted exclusively to a higher density of spindles, whereas other sleep characteristics such as a power of delta band, spindle duration, and general sleep pattern were unaffected. Thus, the present study provides an animal model of learning-dependent sleep changes that can be used for additional electrophysiological investigations.

Cortical spindle activity has indeed been proposed to promote memory-related neuronal plasticity (Steriade et al., 1993; Contreras et al., 1997). Sleep spindles are generated in corticothalamic circuits, which are extensively involved in sensory processing. Their temporal correlation with hippocampal ripples (Siapas and Wilson, 1998) provide a link between hippocampal "episode" traces and neocortical "feature" traces (Pennartz et al., 2002; Kali and Dayan, 2004; Payne and Nadel, 2004). The off-line replay of learning-associated neuronal patterns that are temporally coordinated by brain rhythms can promote the corticothalamic-hippocampal dialogue necessary for memory consolidation (Buzsaki, 1996; Sirota et al., 2003; Moelle et al., 2006; Steriade, 2006). It has been proposed that spindle activity sets the conditions for synaptic changes by favoring calcium entry into the cell and initiation of plasticity-related intracellular cascades (Sejnowski and Destexhe, 2000; Lisman et al., 2002; Benington and Frank, 2003). Indeed, a neuronal firing pattern corresponding to spindle oscillations in vivo has been reported recently to induce synaptic potentiation in neocortical pyramidal cells in vitro (Rosanova and Ulrich, 2005). Thus, increased density of cortical spindles can promote synaptic reinforcement in networks that have been actively engaged during learning.

The off-line replay of learning-associated neuronal patterns during SWS reported in rat studies occur within $1 \mathrm{~h}$ after behavioral experience (Kudrimoti et al., 1999) [but see Louie and Wilson (2001) for REM-associated replay]. This is compatible with our observations of the duration of elevated spindle density. Thus, increased spindle activity may well represent the duration of an initial phase of off-line memory consolidation. It should be noted, however, that neuronal ensemble replay is not limited to the hippocampus; it occurs in the ventral striatum after foraging for reward in a T-maze (Pennartz et al., 2004). Important features of this striatal ensemble replay are that it occurs in close temporal association with hippocampal ripples but it lasts longer than replay in hippocampal neurons (Pennartz et al., 2004). Ribeiro et al. 

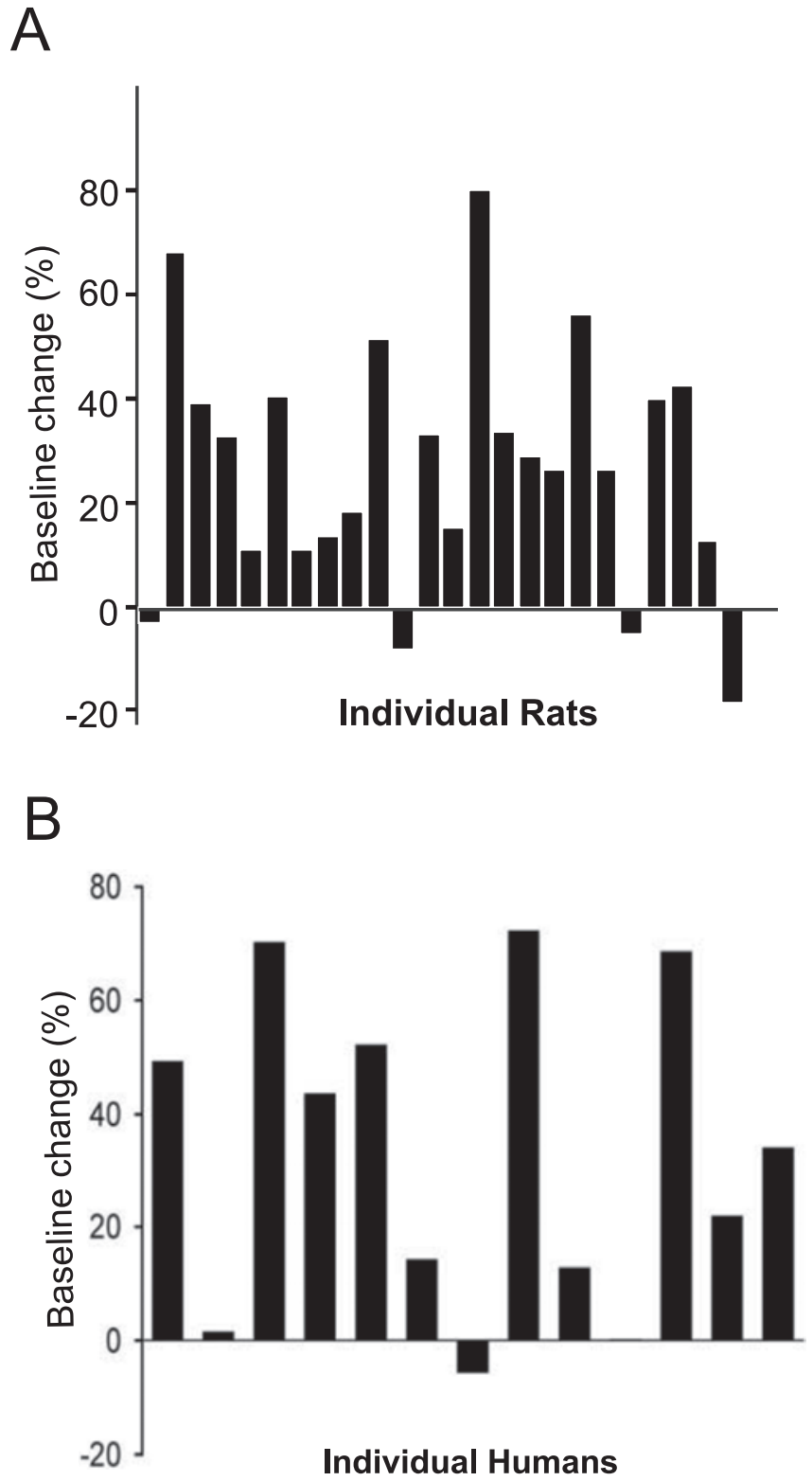

Figure 5. Individual variability of learning-induced elevated spindle density in rats (top; data from experiment 1) and humans (bottom) after a paired-association task. Bars represent the percentage of baseline change for each individual rat or human subject. Human data are modified from Gais et al. (2002).

(2004) have reported "reverberation" of neuronal ensemble activity in multiple forebrain regions lasting up to $48 \mathrm{~h}$ after exploration of a novel object. Despite the fact that the increase in cortical spindle density appears to be limited to the first hour of postlearning sleep, a network reorganization underlying memory consolidation is likely to continue for a longer time. We found a time window during SWS $\sim 2 \mathrm{~h}$ after the rat learned the odorreward contingency when the noradrenergic neurons of the locus ceruleus, which are usually quiescent during SWS, show a surge of activity that is never seen in animals that have not been in the learning situation (Sara et al., 2005). Learning-related delayed release of noradrenaline in forebrain neurons (Tronel et al., 2004), which are potentially engaged in reactivation during sleep, would serve as an effective signal or "molecular switch" for gene expression during a subsequent REM episode (Ribeiro et al., 1999; Cirelli and Tononi, 2000).
The increase in spindle density in the present study was seen after retrieval from remote memory as well as after original learning or extinction. This result lends significant support to the current widely held view that reactivated old memories undergo a "reconsolidation" process to update the information. For the most part, the existence of reconsolidation processes have only been inferred from the fact that amnesia can be induced after reactivation of well established memories (Przybyslawski and Sara, 1997; Nader et al., 2000) (for review, see Sara, 2000; Nader, 2003; Dudai, 2006). If the learning-dependent increase in spindle density is, as it is purported to be, a physiological marker of off-line memory consolidation, then the increase seen after retrieval from remote memory may indeed indicate that a reconsolidation process is taking place. The question remains as to whether this retrieval-related increase in spindle density can be demonstrated in human subjects. In any case, the present results support the view that integration of information across neural networks in different brain regions is critical for memory update after its retrieval.

In conclusion, it is clear from both human and animal studies that there is a great deal of memory processing occurring off-line during sleep. Carefully designed experiments in humans have already revealed relationships between specific types of memory and different sleep stages (Rauchs et al., 2005). These studies have led to much speculation about the underlying neurophysiological mechanisms. Questions such as the relationship of cell-assembly replay to spindle activity in the cortex and ripple activity in the hippocampus must be addressed with animal models. Although the cell-assembly replay data are compelling in terms of memory consolidation, there is so far no behavioral evidence that subsequent memory performance is in any way related to the network replay or that the off-line replay is necessary for retention. The challenge lies in combining the rodent, multisite ensemble recording with behavioral protocols sufficiently complex to drive memory consolidation processes during sleep and allow the animal to express updated memory.

\section{References}

Benington JH, Frank MG (2003) Cellular and molecular connections between sleep and synaptic plasticity. Prog Neurobiol 69:71-101.

Bjorvatn B, Fagerland S, Ursin R (1998) EEG power densities $(0.5-20 \mathrm{~Hz})$ in different sleep-wake stages in rats. Physiol Behav 63:413-417.

Buzsaki G (1989) Two-stage model of memory trace formation: a role for "noisy" brain states. Neuroscience 31:551-570.

Buzsaki G (1996) The hippocampo-neocortical dialogue. Cereb Cortex 6:81-92.

Cirelli C, Tononi G (2000) Differential expression of plasticity-related genes in waking and sleep and their regulation by the noradrenergic system. J Neurosci 20:9187-9194.

Clemens Z, Fabo D, Halasz P (2005) Overnight verbal memory retention correlates with the number of sleep spindles. Neuroscience 132:529-535.

Contreras D, Destexhe A, Sejnowski TJ, Steriade M (1997) Spatiotemporal patterns of spindle oscillations in cortex and thalamus. J Neurosci 17:1179-1196.

De Gennaro L, Ferrara M (2003) Sleep spindles: an overview. Sleep Med Rev 7:423-440.

Dudai Y (2004) The neurobiology of consolidations, or, how stable is the engram? Annu Rev Psychol 55:51-86.

Dudai Y (2006) Reconsolidation: the advantage of being refocused. Curr Opin Neurobiol 16:174-178.

Fogel S, Jacob J, Smith C (2002) The role of sleep spindles in simple motor procedural learning. Sleep [Suppl] 25:A279-A280.

Foster DJ, Wilson MA (2006) Reverse replay of behavioural sequences in hippocampal place cells during the awake state. Nature 440:680-683.

Gais S, Born J (2004) Declarative memory consolidation: mechanisms acting during human sleep. Learn Mem 11:679-685. 
Gais S, Molle M, Helms K, Born J (2002) Learning-dependent increases in sleep spindle density. J Neurosci 22:6830-6834.

Hirase H, Leinekugel X, Czurko A, Csicsvari J, Buzsaki G (2001) Firing rates of hippocampal neurons are preserved during subsequent sleep episodes and modified by novel awake experience. Proc Natl Acad Sci USA 98:9386-9390.

Huber R, Ghilardi MF, Massimini M, Tononi G (2004) Local sleep and learning. Nature 430:78-81.

Kali S, Dayan P (2004) Off-line replay maintains declarative memories in a model of hippocampal-neocortical interactions. Nat Neurosci 7:286-294.

Kudrimoti HS, Barnes CA, McNaughton BL (1999) Reactivation of hippocampal cell assemblies: effects of behavioral state, experience, and EEG dynamics. J Neurosci 19:4090-4101.

Lisman J, Schulman H, Cline H (2002) The molecular basis of CaMKII function in synaptic and behavioural memory. Nat Rev Neurosci 3:175-190.

Louie K, Wilson MA (2001) Temporally structured replay of awake hippocampal ensemble activity during rapid eye movement sleep. Neuron 29:146-156.

Meier-Koll A, Bussmann B, Schmidt C, Neuschwander D (1999) Walking through a maze alters the architecture of sleep. Percept Mot Skills 88:1141-1159.

Moelle M, Yeshenko O, Marshall L, Sara SJ, Born J (2006) Hippocampal sharp wave-ripples linked to slow oscillations in rat slow-wave sleep. J Neurophysiol 96:62-70.

Molle M, Marshall L, Gais S, Born J (2002) Grouping of spindle activity during slow oscillations in human non-rapid eye movement sleep. J Neurosci 22:10941-10947.

Nader K (2003) Memory traces unbound. Trends Neurosci 26:65-72.

Nader K, Schafe GE, Le Doux JE (2000) Fear memories require protein synthesis in the amygdala for reconsolidation after retrieval. Nature 406:722-726.

Pavlides C, Winson J (1989) Influences of hippocampal place cell firing in the awake state on the activity of these cells during subsequent sleep episodes. J Neurosci 9:2907-2918.

Payne JD, Nadel L (2004) Sleep, dreams, and memory consolidation: the role of the stress hormone cortisol. Learn Mem 11:671-678.

Pennartz CM, Uylings HB, Barnes CA, McNaughton BL (2002) Memory reactivation and consolidation during sleep: from cellular mechanisms to human performance. Prog Brain Res 138:143-166.

Pennartz CM, Lee E, Verheul J, Lipa P, Barnes CA, McNaughton BL (2004) The ventral striatum in off-line processing: ensemble reactivation during sleep and modulation by hippocampal ripples. J Neurosci 24:6446-6456.

Przybyslawski J, Sara SJ (1997) Reconsolidation of memory after its reactivation. Behav Brain Res 84:241-246.

Qin YL, McNaughton BL, Skaggs WE, Barnes CA (1997) Memory reprocessing in corticocortical and hippocampocortical neuronal ensembles. Philos Trans R Soc Lond B Biol Sci 352:1525-1533.

Rauchs G, Desgranges B, Foret J, Eustache F (2005) The relationships between memory systems and sleep stages. J Sleep Res 14:123-140.

Ribeiro S, Goyal V, Mello CV, Pavlides C (1999) Brain gene expression dur- ing REM sleep depends on prior waking experience. Learn Mem 6:500-508.

Ribeiro S, Gervasoni D, Soares ES, Zhou Y, Lin SC, Pantoja J, Lavine M, Nicolelis MA (2004) Long-lasting novelty-induced neuronal reverberation during slow-wave sleep in multiple forebrain areas. PLoS Biol 2:E24.

Rosanova M, Ulrich D (2005) Pattern-specific associative long-term potentiation induced by a sleep spindle-related spike train. J Neurosci 25:9398-9405.

Sara SJ (2000) Retrieval and reconsolidation: toward a neurobiology of remembering. Learn Mem 7:73-84.

Sara SJ (2006) Consolidation as concept. In: Science of memory: concepts (Roediger HR, Dudai Y, Fitzpatrick SM, eds). Oxford: Oxford UP, in press.

Sara SJ, Lelong J, Yeshenko O (2005) Late "now print” signal from the locus coeruleus for memory consolidation during slow wave sleep. Soc Neurosci Abstr 31:775.10.

Schabus M, Gruber G, Parapatics S, Sauter C, Klosch G, Anderer P, Klimesch W, Saletu B, Zeitlhofer J (2004) Sleep spindles and their significance for declarative memory consolidation. Sleep 27:1479-1485.

Sejnowski TJ, Destexhe A (2000) Why do we sleep? Brain Res 886:208-223.

Siapas AG, Wilson MA (1998) Coordinated interactions between hippocampal ripples and cortical spindles during slow-wave sleep. Neuron 21:1123-1128.

Sirota A, Csicsvari J, Buhl D, Buzsaki G (2003) Communication between neocortex and hippocampus during sleep in rodents. Proc Natl Acad Sci USA 100:2065-2069.

Skaggs WE, McNaughton BL (1996) Replay of neuronal firing sequences in rat hippocampus during sleep following spatial experience. Science 271:1870-1873.

Smith C (1985) Sleep states and learning: a review of the animal literature. Neurosci Biobehav Rev 9:157-168.

Smith C, MacNeill C (1994) Impaired motor memory for a pursuit rotor task following stage 2 sleep loss in college students. J Sleep Res 3:206-213.

Steriade M (2006) Grouping of brain rhythms in corticothalamic systems. Neuroscience 137:1087-1106.

Steriade M, Timofeev I (2003) Neuronal plasticity in thalamocortical networks during sleep and waking oscillations. Neuron 37:563-576.

Steriade M, McCormick DA, Sejnowski TJ (1993) Thalamocortical oscillations in the sleeping and aroused brain. Science 262:679-685.

Sutherland GR, McNaughton B (2000) Memory trace reactivation in hippocampal and neocortical neuronal ensembles. Curr Opin Neurobiol 10:180-186.

Tronel S, Sara SJ (2002) Mapping of olfactory memory circuits: regionspecific c-fos activation after odor-reward associative learning or after its retrieval. Learn Mem 9:105-111.

Tronel S, Feenstra MG, Sara SJ (2004) Noradrenergic action in prefrontal cortex in the late stage of memory consolidation. Learn Mem 11:453-458.

Walker MP, Stickgold R (2006) Sleep, memory, and plasticity. Annu Rev Psychol 57:139-166.

Wilson MA, McNaughton BL (1994) Reactivation of hippocampal ensemble memories during sleep. Science 265:676-679. 